\title{
SOME APPLICATIONS OF THE STONE- WEIERSTRASS THEOREM TO PLANAR RATIONAL APPROXIMATION
}

\author{
STEVEN MINSKER
}

\begin{abstract}
The Stone-Weierstrass theorem is used to prove two general results about algebras of continuous functions, and each of these yields a necessary and sufficient condition for the planar function algebras $R(K)$ and $C(K)$ to be coincident.
\end{abstract}

We begin with a result which slightly extends the Stone-Weierstrass theorem.

THEOREM 1. (Almost selfadjoint algebras are selfadjoint.) Let $X$ be a compact Hausdorff space, and let $f \in C(X)$. Suppose $m$ and $n$ are relatively prime positive integers. Let $A$ be the closed subalgebra of $C(X)$ generated by $f^{m}, \bar{f}^{n}$, and the constant functions. Then $A=\{g \circ f: g \in C(f(X))\}$. In particular, if $f$ separates the points of $X$, then $A=C(X)$.

Proof. Let $K=f(X)$ and let $B$ be the closed subalgebra of $C(K)$ generated by $z^{m}, \bar{z}^{n}$, and the constant functions. We claim $B=C(K)$. By the usual Stone-Weierstrass theorem, it suffices to show that $z \in B$ and $\bar{z} \in B$. Now $z^{m} \in B$ and $\bar{z}^{n} \in B$ imply $\left(z^{m}\right)^{n}\left(\bar{z}^{n}\right)^{m} \in B$, or $|z|^{2 m n} \in B$. Since the function $h(u)=u^{1 / 2 m n}$ is uniformly approximable by polynomials on the interval $\left[0,\|z\|_{K}^{2 m n}\right]$ and $B$ is closed, it follows that $|z| \in B$. Since $(m, n)=1$, we can find integers $a$ and $b$ with $a m+b n=1$. Without loss of generality, we can assume that $a \geqslant 0, b \leqslant 0$. Then on $K-\{0\}$ we have

$$
z=z^{a m+b n}=\left(z^{m}\right)^{a} /\left(z^{n}\right)^{-b}=\left(z^{m}\right)^{a}\left(\bar{z}^{n}\right)^{-b} /|z|^{-2 b n}
$$

and

$$
\bar{z}=z^{n} / \bar{z}^{n-1}=\bar{z}^{n} z^{n-1} /|z|^{2 n-2} .
$$

For each $\varepsilon>0$, let

$$
h_{\varepsilon}(u)= \begin{cases}1 / \varepsilon^{-2 b n} & \text { on }[0, \varepsilon] \\ 1 / u^{-2 b n} & \text { on }\left[\varepsilon,\|z\|_{K}\right]\end{cases}
$$

Received by the editors September 25, 1975 and, in revised form, December 1, 1975.

AMS (MOS) subject classifications (1970). Primary 46J10; Secondary 46J30.

Key words and phrases. Stone-Weierstrass theorem, rational approximation, dirichlet algebra. 
and let $p_{\varepsilon}(u)$ be a polynomial which uniformly approximates $h_{\varepsilon}(u)$ within $\varepsilon$ on the interval $\left[0,\|z\|_{K}\right]$. Consider the function $q_{\varepsilon}(z)=\left(z^{m}\right)^{a}\left(\bar{z}^{n}\right)^{-{ }^{-} b} p_{\varepsilon}(|z|)$. Clearly $q_{\varepsilon} \in B$. If $z_{0} \in K$ and $\left|z_{0}\right|<\varepsilon$, then

$$
\begin{aligned}
\left|q_{\varepsilon}\left(z_{0}\right)-z_{0}\right| & \leqslant\left|q_{\varepsilon}\left(z_{0}\right)\right|+\left|z_{0}\right| \\
& <\varepsilon^{a m-b n}\left(h_{\varepsilon}\left(\left|z_{0}\right|\right)+\varepsilon\right)+\varepsilon=2 \varepsilon+\varepsilon^{a m-b n+1} .
\end{aligned}
$$

If $z_{0} \in K$ and $\left|z_{0}\right| \geqslant \varepsilon$, then

$$
\begin{aligned}
\left|q_{\varepsilon}\left(z_{0}\right)-z_{0}\right| & =\left|\left(z_{0}^{m}\right)^{a}\left(\bar{z}_{0}^{n}\right)^{-b}\right| \cdot\left|p_{\varepsilon}\left(\left|z_{0}\right|\right)-h_{\varepsilon}\left(\left|z_{0}\right|\right)\right| \\
& <\varepsilon\|z\|_{K}^{a m-b n} .
\end{aligned}
$$

Hence

$$
\left\|q_{\varepsilon}-z\right\|_{K} \rightarrow 0
$$

as $\varepsilon \rightarrow 0$, and so $z \in B$. In completely analogous fashion, if we let

$$
\tilde{h}_{\varepsilon}(u)= \begin{cases}1 / \varepsilon^{2 n-2} & \text { on }[0, \varepsilon], \\ 1 / u^{2 n-2} & \text { on }\left[\varepsilon,\|z\|_{K}\right],\end{cases}
$$

let $\tilde{p}_{\varepsilon}(u)$ be a polynomial which uniformly approximates $\tilde{h}_{\varepsilon}(u)$ within $\varepsilon$ on the interval $\left[0,\|z\|_{K}\right]$, and put $\tilde{q}_{\varepsilon}(z)=\bar{z}^{n} z^{n-1} \tilde{p}_{\varepsilon}(|z|)$, then $\tilde{q}_{\varepsilon} \in B$ and $\left\|\tilde{q}_{\varepsilon}-\bar{z}\right\|_{K}$ $\rightarrow 0$ as $\varepsilon \rightarrow 0$, so $\bar{z} \in B$. This establishes our claim that $B=C(K)$. Since any $g \in C(K)$ is thus uniformly approximable on $K$ by polynomials in $z^{m}$ and $\bar{z}^{n}$, it follows that $g \circ f$ is uniformly approximable on $X$ by polynomials in $f^{m}$ and $\bar{f}^{n}$. Hence $A \supset\{g \circ f: g \in C(f(X))\}$. The remaining assertions are obvious.

COROllary. Let $K$ be a compact subset of the complex plane. Then $R(K)$ $=C(K)$ iff $\bar{z}^{n} \in R(K)$ for some $n \in \mathbf{N}$.

Proof. Immediate from the theorem.

REMARK. An analysis of the proof of Theorem 1 shows that the closed subalgebra of $C(X)$ generated by $f^{m}$ and $\bar{f}^{n}$ contains both $f$ and $\bar{f}$; that is, $\left\{f^{m}, \bar{f}^{n}\right\}$ and $\{f, \bar{f}\}$ generate the same closed subalgebra of $C(X)$. Consequently, the following extended Stone-Weierstrass theorem holds:

Let $X$ be a compact Hausdorff space and let $A$ be a subalgebra of $C(X)$ such that:

(1) if $x_{0} \in X$, then there exists $f \in A$ with $f\left(x_{0}\right) \neq 0$,

(2) A separates the points of $X$,

(3) if $f \in A$, then $\bar{f}^{n} \in A$ for some $n \in \mathbf{N}$ depending on $f$.

Then $A$ is dense in $C(X)$.

Theorem 2. Let $X$ be a compact Hausdorff space, and let $f \in C(X)$. Let $\alpha>0$. If $A$ is the subalgebra of $C(X)$ generated by $f,|f|^{\alpha}$, and the constant functions, then $\operatorname{Re} A$ (i.e., the real parts of functions in $A$ ) is dense in 
$\left\{g \circ f: g \in C_{\mathbf{R}}(f(X))\right\}$. In particular, if $f$ separates the points of $X$, then $A$ is a dirichlet algebra on $X$ (i.e., $\operatorname{Re} A$ is dense in $C_{\mathbf{R}}(X)$ ).

Proof. Let $K=f(X)$, and let $B$ be the closed subalgebra of $C(K)$ generated by $z,|z|^{\alpha}$, and the constant functions. We claim $\operatorname{Re} B$ is dense in $C_{\mathbf{R}}(K)$. As in the proof of Theorem $1,|z|^{\alpha} \in B$ implies $|z| \in B$. If $j$ and $k$ are nonnegative integers with $j \geqslant k$, then $\operatorname{Re}\left(z^{j} \bar{z}^{k}\right)=\operatorname{Re}\left(z^{j-k}|z|^{2 k}\right) \in \operatorname{Re} B$, and if $j<k$, then

$$
\operatorname{Re}\left(z^{j} \bar{z}^{k}\right)=\operatorname{Re}\left(\overline{z^{j} \bar{z}^{k}}\right)=\operatorname{Re}\left(z^{k} \bar{z}^{j}\right)=\operatorname{Re}\left(z^{k-j}|z|^{2 j}\right) \in \operatorname{Re} B .
$$

Analogously, $\operatorname{Im}\left(z^{j} \bar{z}^{k}\right)=\operatorname{Re}\left(-i z^{j} \bar{z}^{k}\right)$ implies that $\operatorname{Im}\left(z^{j} \bar{z}^{k}\right) \in \operatorname{Re} B$. By the Stone-Weierstrass theorem, the algebra generated by $z, \bar{z}$, and the constants is dense in $C(K)$. The density of $\operatorname{Re} B$ in $C_{\mathbf{R}}(K)$ follows immediately, establishing our claim. Since any $g \in C_{\mathbf{R}}(K)$ is thus uniformly approximable on $K$ by the real parts of polynomials in $z$ and $|z|^{\alpha}, g \circ f$ is uniformly approximable on $X$ by the real parts of polynomials in $f$ and $|f|^{\alpha}$. This completes the proof.

Corollary. Let $K$ be a compact subset of the complex plane. Then $R(K)$ $=C(K)$ iff $|z|^{\alpha} \in R(K)$ for some $\alpha>0$.

Proof. By the preceding theorem, $|z|^{\alpha} \in R(K)$ implies that $R(K)$ is a dirichlet algebra on $K$. This in turn implies that every point of $K$ is a peak point for $R(K)$. By a theorem of Bishop [1, Theorem 3.3.3], we conclude $R(K)=C(K)$.

\section{REFERENCES}

1. A. Browder, Introduction to function algebras, Benjamin, New York, 1969. MR 39 \#431.

Department of Mathematics, University of Pittsburgh, Pittsburgh, Pennsylvania 15260 Current address: Department of Mathematics, Rutgers University, Camden, New Jersey 08102 